\title{
Relationship between training load and match running performance in men's soccer
}

\author{
Rui Silva ${ }^{1}$, Miguel Camões ${ }^{1,2}$, António Barbosa ${ }^{1}$, Georgian Badicu ${ }^{3, \star}$, Hadi Nobari ${ }^{4,5,6}$, \\ André Bernardo ${ }^{1}$, Sílvio Afonso Carvalho ${ }^{7}$, Tiago Sant'Ana ${ }^{1}$, Ricardo Lima ${ }^{1,2}$, \\ Pedro Bezerra1, Filipe Manuel Clemente ${ }^{1,8}$ \\ ${ }^{1}$ Escola Superior Desporto e Lazer, Instituto Politécnico de Viana do Castelo, Rua Escola Industrial e Comercial de Nun'Álvares, \\ 4900-347 Viana do Castelo, Portugal \\ ${ }^{2}$ The research Centre in Sports Sciences, Health Sciences and Human Development (CIDESD), 5001-801 Vila Real, Portugal \\ ${ }^{3}$ Department of Physical Education and Special Motricity, University Transilvania of Brasov, 500068 Brasov, Romania \\ ${ }^{4}$ Department of Physical Education and Sports, University of Granada, 18009 Granada, Spain \\ ${ }^{5}$ Department of Exercise Physiology, Faculty of Sport Sciences, University of Isfahan, 81746-7344 Isfahan, Iran \\ ${ }^{6}$ HEME Research Group, Faculty of Sport Sciences, University of Extremadura, 10003 Cáceres, Spain \\ ${ }^{7}$ Associação de Futebol de Bragança, 5300-861 Bragança, Portugal \\ ${ }^{8}$ Instituto de Telecomunicações, Delegação da Covilhã, 1049-001 Lisboa, Portugal
}

*Correspondence: georgian.badicu@unitbv.ro (Georgian Badicu)

\begin{abstract}
Objectives: The main aim of this study was to test the relationship of volume and weekly training intensities with match-running demands of professional male soccer players.

Methods: The training volume and intensity load and match demands of 18 professional soccer players (age: $20.7 \pm 1.8$ years) were monitored daily for 15 weeks.

Results: Match high-speed running (mHSR) and match accelerations (mACC) were found to be moderately positively correlated with weekly HSR (wtHSR) and weekly ACC volume (wtACC) ( $r=0.497$; $p<0.01 ; r=0.367 ; p<0.01)$, respectively. Moderate positive correlations were also found between mHSR, mACC and mHSR and mean training intensity of ACC (mtACC) $(r=0.366 ; p<0.01)$.

Conclusions: Weekly HSR and ACC training volume and intensity have moderate associations with match HSR and ACC loads. Coaches should prioritize weekly HSR and ACC during the training process, for ensuring that players perform more HSR and ACC actions during the subsequent match.
\end{abstract}

\section{Keywords}

Football; Athletic performance; Performance analysis; Sports training

\section{Introduction}

The quantification of professional male soccer players' running demands during training and competitions has gained an enormous amount of interest from sports scientists and coaches [1]. From a team sports perspective, soccer is documented as being one of the most demanding sports in terms of total distance (TD) covered [2]. Indeed, several studies have analyzed soccer match external load demands [3-5]. From the above-mentioned studies, it was demonstrated that match external loads are influenced by different playing positions and tactical formations [5]. During a training week, greater loads were observed in match, and lower loads were observed in the day before match, with session-rate of perceived exertion (s-RPE) values declining during the three days before match [4]. It was previously shown that daily s-RPE training loads vary between 15 and 111 A.U., and weekly s-RPE values vary between 259 and 350 A.U., depending on the season period and playing position [4, 6]. Furthermore, it has been documented that during a soccer match players can reach approximately $11.000 \mathrm{~m}$ of total distance (TD), $900 \mathrm{~m}$ of high-speed running (HSR), $2472 \mathrm{~m}$ of high-metabolic load 
(HML), $61 \mathrm{n}$ of accelerations (ACC) $\left(\geq 3 \mathrm{~m} \cdot \mathrm{s}^{2}\right)$ and 58 of decelerations (DEC) $\left(\geq 3 \mathrm{~m} \cdot \mathrm{s}^{2}\right)[7,8]$. The relationship between accumulated weekly training load and match demands can vary in accordance to the nature of each external load measure. Given that, a proper understanding of accumulated training loads and match demands are crucial to adjust the training process and ensure correct progressions of the load imposed on athletes [4].

The monitoring of the external loads imposed on athletes are part of the monitoring cycle framework proposed by Gabbett et al. [9]. This proposal is comprised of four main objective and subjective dimensions, which englobes the monitoring of external and internal loads, wellness, and readiness to train. In the specific case of subjective internal load measures, the use of s-RPE has been proved to be a great tool for internal load quantification [10]. The s-RPE is calculated by multiplying the score reported on a 10-point scale of exertion by the duration of the training session (in s) $[11,12]$. Furthermore, considering the relevance of assessing the current state of thletes for training regulation, the abovementioned practices can assist coaches in monitoring weekly training and competition loads [13].

Training load quantification allows practitioners to monitor whether important training principles, such as progressive overload, individualization, and variation, are being ensured [14, 15]. Studies on this topic focus on two main categories of training load quantification: (i) external load (physical demands imposed by training tasks) and (ii) internal load (biological responses to a given external stimulus) [16]. In male team sports, the most commonly used methods for quantifying external loads are global positioning systems (GPS), accelerometers, and inertial measurement units, the latter two of which are usually embedded in modern GPS devices [17, 18]. The common GPS metrics used in soccer are distance-based measures covered at different speed thresholds (arbitrarily defined velocities $\left[\mathrm{km} \cdot \mathrm{h}^{-1}\right]$ for each metric), accelerometry-based measures such as HML (distances covered at a speed of $>5.5 \mathrm{~m} \cdot \mathrm{s}^{2}$ while accelerating and decelerating), and actions related to changes in velocity ACC $\geq 3 \mathrm{~m} \cdot \mathrm{s}^{2}$ and DEC $\geq 3 \mathrm{~m} \cdot \mathrm{s}^{2}[19-21]$.

Recently, research has analyzed the accumulated weekly external training loads (both using distanceand accelerometry-based metrics) and their related workload indices [22, 23]. Also, other research has focused on analyzing weekly training (wt) external loads relative to match loads [7, 24]. For instance, Stevens et al. [7] revealed that weekly TD, HSR, ACC, and DEC $\left(\geq 3 \mathrm{~m} \cdot \mathrm{s}^{2}\right)$ were lower than corresponding match loads. Also, ACC and DEC seem to be more closely related to match loads than other training load metrics [7]. In fact, that study which was conducted on professional soccer players from the English Premier League, revealed that soccer players can reach approximately 20.000 $\mathrm{m}$ of TD, and $440 \mathrm{~m}$ of HSR during a training week [25]. Also, another study conducted on Dutch professional soccer players revealed weekly HML distances of approximately $3848 \mathrm{~m}, 181 \mathrm{n}$ of ACC $\left(\geq 3 \mathrm{~m} \cdot \mathrm{s}^{2}\right)$ and $131 \mathrm{n}$ of DCC $(\geq 3$ $\left.\mathrm{m} \cdot \mathrm{s}^{2}\right)$ [7]. Although there are some studies comparing accumulated training load relative to match load, there are a lack of studies analyzing possible associations between mean training load intensity and match running performance in soccer $[7,24]$. In fact, one of the few studies on this topic revealed that match running performance metrics, especially TD and HSR, had strong associations with the previous weekly training workload volume [26]. However, that study did not directly test the relationships between training intensity with match loads.

Some distance-based metrics can depend on tactical characteristics and other contextual factors [26, 27]. Also, accelerometry-based measures seem to be useful for monitoring neuromuscular status [28]. Given the aforementioned evidence, the aim of this study is to test the relationships of weekly training volumes and intensities with match-running demands in professional male soccer players.

\section{Methods}

\subsection{Experimental approach to the problem}

An observational analytic cohort design was conducted in this study. A correlational study design was conducted to test possible relationships between weekly training load (volume and intensity) and the load intensity of a match during the same week. For each of the analyzed weeks, only the players participating in all training sessions and the match were included (Table 1).

T A B L E 1. Eligible players for each analyzed week.

\begin{tabular}{lcc}
\hline & Training sessions (N) & Players eligible $(\mathrm{N})$ \\
\hline Week 6 & 5 & 11 \\
Week 8 & 5 & 12 \\
Week 10 & 5 & 12 \\
Week 11 & 5 & 13 \\
Week 13 & 5 & 11 \\
Week 14 & 6 & 12 \\
Week 16 & 5 & 10 \\
Week 17 & 5 & 10 \\
Week 18 & 5 & 9 \\
Week 22 & 4 & 10 \\
Week 23 & 6 & 9 \\
Week 26 & 5 & 8 \\
Week 27 & 4 & 11 \\
Week 28 & 5 & 7 \\
Week 29 & 5 & 10 \\
\hline
\end{tabular}

The volume of weekly training load (wt) represents the sum of all accumulated load demands in all the training sessions of any given week. The intensity of the training sessions represents the mean intensity of all training (mt) sessions. Thus, the volume represents the total number of meters covered or the total number of actions performed in a week; meanwhile, intensity represents the mean number of meters covered per min of training or the number of actions made per min of training. For matches, only the intensity was considered, with the aim to standardize the playing time of each player. The period of observation corresponded to 
microcycles six to twenty-nine of the season.

\subsection{Participants}

Eighteen professional male soccer players (age: $20.7 \pm 1.8$ years; body mass: $69.8 \pm 5.9 \mathrm{~kg}$; height: $179.2 \pm 5.0 \mathrm{~cm}$; years of experience: $11.0 \pm 2.8$ ) participated in the study. All players belonged to the same club, competing in the 3rd Portugal league. During the observational period, the team had four-to-six training sessions per week, with an average time of session of $78.8 \pm 15.6 \mathrm{~min}$, and with one official match per week. For each week, the inclusion criteria in this study was: (i) the player was involved in all the training sessions and in the match; (ii) the player was not injured or ill in the week preceding match participation. The exclusion criteria was: (i) did not participate in all training sessions the week before the match; (ii) did not participate in match. All players were informed regarding the study design, protocol, benefits and the risks of study participation. All athletes signed an informed consent in which was explicit that they were free to give up on the process at any time. The study protocol was approved by a scientific council of the local university (CTC-ESDL-CE002-2021) and followed the ethical standards of Declaration of Helsinki for the study in humans.

\subsection{Rate of perceived exertion}

For internal load quantification, 10 to $30 \mathrm{~min}$ after each training session and match, the subjective rate of perceived exertion (RPE) values were collected as previously documented [29]. To monitor players' RPE, the CR-10 method was used [12]. Based on CR-10 method, a value of 1 refers to "very light activity" and 10 refers to "maximal exertion". Each player attributed RPE values individually, without the influence of their colleagues. All athletes used a smartphone to answer the question "how hard was your training?" in an online survey. All the players were previously familiarized to this kind of perceptual effort rating. The internal training loads were then obtained by multiplying each player RPE score by each training session duration in $\min [30]$.

\subsection{External load}

Every athlete used the same 18-Hz GPS (STATSports, Apex, Northern Ireland) during the observational period. The GPS units have an integrated $100-\mathrm{Hz}$ gyroscope, $100-\mathrm{Hz}$ tri-axial accelerometer and $10-\mathrm{Hz}$ magnetometer. Also, the GPS model used was previously tested for its validity and reliability [31, 32]. Typical error as coefficient of variation was $1.6 \%$ at $400-\mathrm{m}$ distance and $0.7 \%$ at $20-\mathrm{m}$ peak speed [32]. The GPS units were placed in a specific vest in which the unit was fixed between the scapulae. The data collected during training sessions and matches were imported and processed in the STATSports Sonra software (version 3.0, STATSports, Apex, Northern Ireland).

The GPS measures were collected daily during each training session and match, and were comprised of the following: (i) total distance (TD: consisting in the total distance covered by players); (ii) distance covered at high-speed running (HSR: distances covered at a speed of $19.8 \mathrm{~km} \cdot \mathrm{h}^{-1}$ or above); (iii) high metabolic load distances (HML: distances covered at a speed of $>19.8 \mathrm{~km} \cdot \mathrm{h}^{-1}$ while accelerating/decelerating at $\geq 2 \mathrm{~m} \cdot \mathrm{s}^{2}$ ); (iv) high intensity accelerations and decelerations (ACC and DEC: number of accelerations and decelerations at $\left.\geq 3 \mathrm{~m} \cdot \mathrm{s}^{2}\right)[31]$.

\subsection{Statistical procedures}

Descriptive statistics were presented in forms of tables, using mean and standard deviation. Normality of the sample was observed using Kolmogorov-Smirnov test $(p>0.05)$. Pearson $r$ correlation test $(r)$ was executed to determine the relationships between training load (volume and intensity) and match-running intensity measures. Magnitude of correlations were interpreted using the following thresholds: 0.00.1 , trivial; 0.1-0.3, small; 0.3-0.5, moderate; 0.5-0.7, large; $0.7-0.9$, very large; $>0.9$, nearly perfect [33]. The statistical procedures were executed on SPSS software (version 25.0, IBM, Armonk, NY, USA) for a $p<0.05$. The forest plots representing the relationship between variables have reported the $\mathrm{r} 2$, which represents the square of the sample correlation coefficient.

\section{Results}

Descriptive statistics of training load (volume and intensity) and match running demands over the period of observation can be found in Table 2 .

Relationships between volume of training load (sum of weekly training load for different measures) and matchrunning intensity can be found in Table 3. Moderate correlations were found between mHSR and wtHSR ( $\mathrm{r}=$ 0.497; $p<0.01$ ). Also, moderate correlations were found between mACC and wtACC $(\mathrm{r}=0.367 ; p<0.01)$

Relationships between intensity of training load (mean of weekly training load intensity for different measures) and match-running intensity can be found in Table 4. Moderate correlations were found between mHSR and mtHSR $(\mathrm{r}=$ $0.498 ; p<0.01)$ and between $\mathrm{mACC}$ and $\mathrm{mtACC}(\mathrm{r}=0.366$; $p<0.01)$.

Fig. 1 presents the scatterplot for the relationships between mHSR and wtHSR $\left(R^{2}=0.247\right)$ and $m$ tHSR $\left(R^{2}=0.249\right)$.

\section{Discussion}

The purpose of this study was to analyze the relationships of weekly training volume and intensity with match external measures relativized per min, in professional male soccer players. The primary findings revealed that both wtHSR and wtACC had moderate positive relationships with match relative mHSR and mACC demands.

First, the weekly training volume and intensity of TD and HSR reported in the present study are in concordance with other research conducted on Dutch professional male soccer players [7]. In fact, the study of Stevens et al. [7], revealed that wt TD and wtHSR were $22454 \pm 1444 \mathrm{~m}$ and $811 \pm 78 \mathrm{~m}$, 
T A B L E 2. Descriptive statistics for weekly training load, weekly intensity training load and match intensity load.

\begin{tabular}{lcccccc}
\hline Volume training & wtTD $(\mathrm{m})$ & wtHSR $(\mathrm{m})$ & wtACC $(\mathrm{n})$ & wtDEC $(\mathrm{n})$ & wtHML (A.U.) & wtsRPE (A.U.) \\
\hline Mean \pm SD & $23126 \pm 3882$ & $1166 \pm 437$ & $274 \pm 67$ & $235 \pm 58$ & $3731 \pm 806$ & $1533 \pm 379$ \\
Intensity training & $\mathrm{mtTD}(\mathrm{m} / \mathrm{min})$ & $\mathrm{mtHSR}(\mathrm{m} / \mathrm{min})$ & $\mathrm{mtACC}(\mathrm{n})$ & $\mathrm{mtDEC}(\mathrm{n})$ & $\mathrm{mtHML}($ A.U.) & $\mathrm{mtsRPE}($ A.U.) \\
Mean $\pm \mathrm{SD}$ & $61.9 \pm 5.7$ & $3.07 \pm 1.07$ & $0.73 \pm 0.14$ & $0.62 \pm 0.12$ & $9.85 \pm 1.59$ & $306.3 \pm 63.4$ \\
Match intensity & $\mathrm{mTD}(\mathrm{m} / \mathrm{min})$ & $\mathrm{mHSR}(\mathrm{m} / \mathrm{min})$ & $\mathrm{mACC}(\mathrm{n} / \mathrm{min})$ & $\mathrm{mDEC}(\mathrm{n} / \mathrm{min})$ & $\mathrm{mHML}($ A.U./min $)$ & $\mathrm{msRPE}$ (A.U.) \\
Mean $\pm \mathrm{SD}$ & $95.3 \pm 32.0$ & $6.59 \pm 3.41$ & $0.85 \pm 0.38$ & $0.87 \pm 0.35$ & $18.4 \pm 6.89$ & $566.0 \pm 214.4$ \\
\hline
\end{tabular}

wt, weekly training load (accumulated load of the sessions previous to the match); $\mathrm{mt}$, mean training load (mean of intensity in weekly training sessions); TD, total distance; HSR, high speed running; ACC, accelerations; DEC, decelerations; HML, high metabolic load; sRPE, sessionRPE; n, number; m, meters; min, minutes; A.U., arbitrary units, SD, standard-deviation.

T A B L E 3. Correlations (mean and $95 \%$ confidence interval) between weekly training load and the intensity of match-running demands.

\begin{tabular}{lcccccc}
\hline & wtTD $(\mathrm{m})$ & wtHSR $(\mathrm{m})$ & wtACC $(\mathrm{n})$ & wtDEC $(\mathrm{n})$ & wtHML $($ A.U. $)$ & wtsRPE $($ A.U. $)$ \\
\hline mTD (m/min) & $0.155(0.16 ; 0.31)$ & $0.132(-0.03 ; 0.28)$ & $0.099(-0.06 ; 0.25)$ & $0.172^{*}(0.01 ; 0.32)$ & $0.163^{*}(0.01 ; 0.31)$ & $0.073(-0.09 ; 0.23)$ \\
mHSR (m/min) & $0.172(0.01 ; 0.32)$ & $0.497^{* *}(0.37 ; 0.61)$ & $0.143(-0.01 ; 0.29)$ & $0.175^{*}(0.02 ; 0.32)$ & $0.261^{* *}(0.11 ; 0.40)$ & $0.098(-0.06 ; 0.25)$ \\
mACC (n/min) & $0.150(-0.01 ; 0.3)$ & $0.123(-0.04 ; 0.28)$ & $0.367^{* *}(0.22 ; 0.50)$ & $0.291^{* *}(0.14 ; 0.43)$ & $0.244^{* *}(0.09 ; 0.39)$ & $0.151(-0.01 ; 0.30)$ \\
mDEC (n/min) & $0.077(-0.08 ; 0.23)$ & $0.206^{* *}(0.05 ; 0.35)$ & $0.169^{*}(0.01 ; 0.32)$ & $0.254^{* *}(0.10 ; 0.40)$ & $0.195^{*}(0.04 ; 0.34)$ & $0.078(-0.08 ; 0.23)$ \\
mHML (A.U./min) & $0.118(-0.04 ; 0.27)$ & $0.221^{* *}(0.07 ; 0.37)$ & $0.142(-0.02 ; 0.29)$ & $0.203^{*}(0.05 ; 0.35)$ & $0.218^{* *}(0.06 ; 0.36)$ & $0.111(-0.05 ; 0.26)$ \\
msRPE (A.U.) & $-0.076(-0.23 ; 0.8)$ & $-0.114(-0.27 ; 0.04)$ & $-0.077(-0.23 ; 0.08)$ & $-0.067(-0.22 ; 0.09)$ & $-0.060(-0.22 ; 0.10)$ & $-0.122(-0.27 ; 0.04)$ \\
\hline
\end{tabular}

wt, weekly training load (accumulated load of the sessions previous to the match); TD, total distance; HSR, high speed running; ACC, accelerations; DEC, decelerations; HML, high metabolic load; sRPE, session-RPE; n, number; m, meters; min, minutes; A.U., arbitrary units.

* Correlation is significant at the $p<0.05$; $^{* *}$ Correlation is significant at the $p<0.01$.

T A B L E 4. Correlations (mean and $95 \%$ confidence interval) between mean of intensity of training load demands and the intensity of match-running demands.

\begin{tabular}{|c|c|c|c|c|c|c|}
\hline & $\operatorname{mtTD}(\mathrm{m})$ & $\mathrm{mtHSR}(\mathrm{m})$ & $\operatorname{mtACC}(\mathrm{n})$ & $\operatorname{mtDEC}(\mathrm{n})$ & mtHML (A.U.) & mtsRPE (A.U.) \\
\hline $\mathrm{mTD}(\mathrm{m} / \mathrm{min})$ & $0.105(-0.05 ; 0.26)$ & $0.116(-0.04 ; 0.27)$ & $0.049(-0.11 ; 0.21)$ & $0.143(-0.01 ; 0.29)$ & $0.121(-0.04 ; 0.27)$ & $0.024(-0.13 ; 0.18)$ \\
\hline $\operatorname{mHSR}(\mathrm{m} / \mathrm{min})$ & $0.114(-0.04 ; 0.27)$ & $0.498^{* *}(0.37 ; 0.61)$ & $0.095(-0.06 ; 0.25)$ & $0.134(-0.02 ; 0.29)$ & $0.253^{* *}(0.10 ; 0.39)$ & $0.076(-0.08 ; 0.23)$ \\
\hline $\mathrm{mACC}(\mathrm{n} / \mathrm{min})$ & $0.036(-0.12 ; 0.19)$ & $0.082(-0.08 ; 0.24)$ & $0.366^{* *}(0.22 ; 0.50)$ & $0.271^{* *}(0.12 ; 0.41)$ & $0.194^{*}(0.04 ; 0.34)$ & $0.111(-0.05 ; 0.26)$ \\
\hline $\operatorname{mDEC}(\mathrm{n} / \mathrm{min})$ & $0.014(-0.14 ; 0.17)$ & $0.201^{*}(0.04 ; 0.35)$ & $0.171^{* *}(0.01 ; 0.32)$ & $0.283^{* *}(0.13 ; 0.42)$ & $0.193^{*}(0.04 ; 0.34)$ & $0.034(-0.12 ; 0.19)$ \\
\hline mHML (A.U./min) & $0.077(-0.08 ; 0.23)$ & $0.221^{* *}(0.07 ; 0.37)$ & $0.118(-0.04 ; 0.27)$ & $0.197^{*}(0.04 ; 0.34)$ & $0.219^{*}(0.06 ; 0.36)$ & $0.069(-0.09 ; 0.22)$ \\
\hline msRPE (A.U.) & $-0.016(-0.17 ; 0.14)$ & $-0.068(-0.22 ; 0.09)$ & $-0.021(-0.18 ; 0.14)$ & $-0.030(-0.19 ; 0.13)$ & $-0.005(-0.16 ; 0.15)$ & $-0.173^{*}(-0.32 ;-0.02)$ \\
\hline
\end{tabular}

mt, mean training load (mean of intensity in weekly training sessions); TD, total distance; HSR, high speed running; ACC, accelerations; DEC, decelerations; HML, high metabolic load; sRPE, session-RPE; n, number; $m$, meters; min, minutes; A.U., arbitrary units.

* Correlation is significant at the $p<0.05$; ${ }^{* *}$ Correlation is significant at the $p<0.01$.
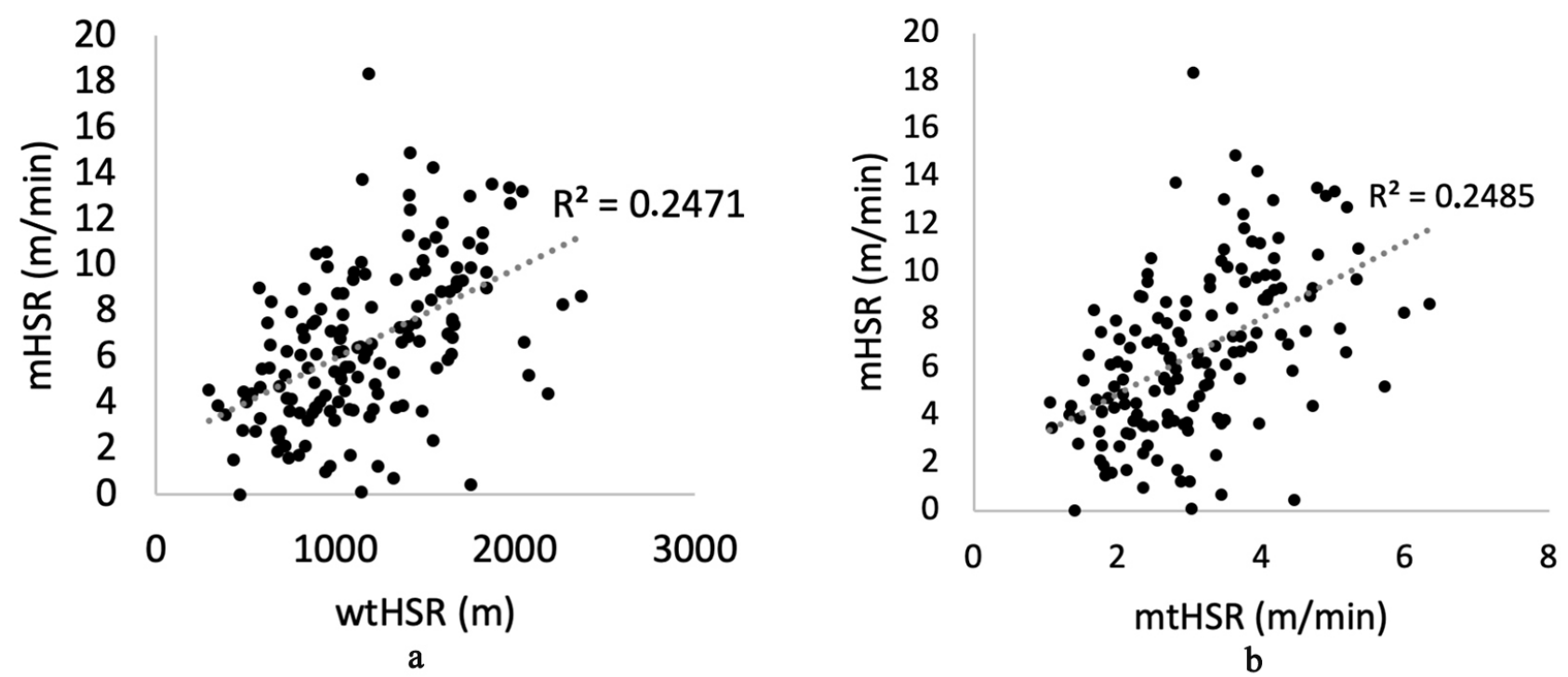

FIG. 1. Scatterplot for relationships between mean training high-speed running (mHSR) load and (a) weekly training high-speed running (wHSR) and (b) mean training high-speed running (mtHSR). 
and their corresponding intensities were $72.6 \pm 7.8$ and $2.6 \pm$ 0.1 , respectively. The authors of the above-mentioned study analyzed only four training sessions before match (match day: MD-4 to MD-1), while in the present study, the overall analyzed weeks had five training sessions before match (MD5 to $\mathrm{MD}-1$ ). However, the training volume and intensity of MD-5 (the day after day-off) are usually low [34]. This may explain why our reported weekly values are similar to the study of Stevens et al. [7], even with one more training session per week. However, other studies have reported higher weekly training volumes for both distance-based and accelerometry-based metrics than those used in the present study [22, 23]. These discrepancies might be due to the number of weekly sessions analyzed in each study, as the speed thresholds in our study are the same as those used in the three aforementioned studies [7, 22, 23]. Indeed, one study only analyzed four weekly training sessions [7]. On the other hand, despite the overall analyzed weeks of the other two above-mentioned studies had five training sessions, the authors considered the absolute match demands as part of weekly accumulated load. In contrast, in the present study, the weekly accumulated load was comprised of training days only (from MD-5 to MD-1). The match loads were not considered as part of weekly load.

Regarding the relationships between weekly training volume and intensity with match-running demands in professional male soccer players, it was observed that mHSR and mACC measures increased when HSR and ACC training volume and intensity increased. However, thresholds are yet to be determined. A recent study conducted on 18 professional male soccer players from the English Championship revealed that prior training workloads had trivial-to-moderate relationships with subsequent match performance in terms of high-intensity actions such as HSR, ACC, DEC, and HML distances [35]. The present study also revealed similar relationships to the study of Springham et al. [35], between prior weekly training volume and subsequent relative HSR and ACC match physical demands, but not for DEC and HML measures. However, that study only tested the relationships between weekly training volume and match intensity. The lack of significant relationships in the present study, between both DEC and HML weekly training volume and intensity and relative match demands for those measures, might be due to other contextual factors such as the quality of opposition during official matches, winning or losing, fatigue, tactical determinants, among others [36]. Interestingly, also considering the weekly accumulated training loads and match loads, Clemente et al. [24] revealed only trivial-to-small correlations between them, suggesting that soccer training loads are independent of subsequent match loads, due to the above-mentioned contextual factors.

Notwithstanding the above discussed relationships between training volume and relative match demands, our results revealed positive moderate relationships between prior training workload intensity and relative match demands. In fact, another study on 56 male professional soccer players from two different teams confirmed that the physical performance exhibited during a match could be predicted based on the training workload volume of the week leading up to the match [26]. Our findings are in contrast with the above-mentioned study which revealed that mTD and mHSR had strong associations with the previous wtTD and wtHSR loads - in terms of wtTD. Also, the same authors [26] suggested that may be preferable to maintain training intensity high while maintaining training volumes low. This may be reflected by lower total distances covered and higher high-intensity activity during training. The differences in speed thresholds for each GPS metric used between our study and the above-mentioned study, can make comparisons with our results difficult [20]. Considering that both HSR and ACC weekly training volume and intensity were associated with mHSR and mACC performance in the present study, it would be beneficial for soccer athletes to be exposed to HSR and ACC demands similar to those observed during matches.

It was previously documented that TD and the number high ACC during the training week are three-to-four times greater than the match demands, and that HSR are one-totwo times greater [24]. The same authors attributed those findings to the fact that Portuguese coaches use small-sided games as a training philosophy which causes an higher frequency of ACC and lower of HSR. In fact, a study conducted on twelve elite soccer players from the English Premier League revealed that the majority of weekly training distances covered were comprised of low-intensity activities, while the the majority of high-intensity distances were completed during the match [8]. This may suggest that soccer training focusing more on volume than intensity might not prepare the athletes for match worst-case scenario [37]. Since soccer is an intermittent sport characterized by high-intensity activity, it would be important to ensure a progressive overload on high-intensity demands without severely increasing the training volume in terms of wtTD [38]. Coaches must ensure that athletes are exposed to moderate weekly highintensity activity to better cope with match demands [39, 40].

This study has its limitations. One of the main limitations was related to the small sample size, which makes it difficult to generalize the results. This is a very common issue among studies conducted in professional team settings. Also, we did not analyze weekly training and match loads in a continuous fashion, which may interfere with the inferences made in the present study. However, this issue was inevitable, as we did not consider the congested weeks (with more than one match in the same week) of the analyzed team for the analysis. Finally, the players included in the weeks analyzed were not always the same, since they were selected or not for the match based on coaches selection. This may represent an issue in heterogeneity from week to week analysis. Future studies should be conducted on congested fixtures to analyze the effects of training volume and intensity with the matches in between.

\section{Conclusions}

The main findings of the present study revealed that weekly HSR and ACC training volume and intensity had moderate 
relationships with match relative HSR and ACC measures. During a match, HSR and ACC measures increase when the weekly accumulated loads and average training intensity of both measures are increased. Given that HSR and ACC are important match performance determinants, coaches should prioritize these training measures during weekly training.

\section{Abbreviations}

TD, total distance; HSR, high-speed running; ACC, accelerations; DEC, decelerations; GPS, global positioning system; IMU, inertial measurement units; RPE, rate of perceived exertion; s-RPE, session-rate of perceived exertion; $\mathrm{mt}$, mean training load (mean of intensity in weekly training sessions); wt, weekly training load (accumulated load of the sessions previous to the match); HML, high metabolic load; n, number; m, meters; min, minutes; A.U., arbitrary units.

\section{Author contributions}

RS conceived and designed the experiments. FMC conceived, designed and supervised the experiments. RS, MC, ABar, GB, HN, ABer, SAC, TS, RL, PB and FMC written, revised and approved the article.

\section{Ethics approval and consent to participate}

All procedures performed in studies involving human participants were in accordance with the ethical standards of institutional and/or national research committee and with the 1964 Helsinki declaration and its later amendments or comparable ethical standards. Informed consent was obtained from all individual participants included in the study. The study protocol was approved by a scientific council of the local university (CTC-ESDL-CE002-2021) and followed the ethical standards of Declaration of Helsinki for the study in humans.

\section{Acknowledgment}

This study was done as part of a master thesis in sports training, Escola Superior de Desporto e Lazer, Instituto Politécnico de Viana do Castelo, Portugal.

\section{Funding}

This research received no external funding.

\section{Conflict of interest}

The authors declare no conflict of interest.

\section{References}

[1] Miguel M, Oliveira R, Loureiro N, García-Rubio J, Ibáñez SJ. Load measures in training/match monitoring in soccer: A systematic review. International Journal of Environmental Research and Public Health. 2021; 18: 1-26.

[2] Taylor JB, Wright AA, Dischiavi SL, Townsend MA, Marmon
AR. Activity Demands during Multi-Directional Team Sports: a Systematic Review. Sports Medicine. 2017; 47: 2533-2551.

[3] Clemente FM, Couceiro MS, Martins FML, Ivanova MO, Mendes R. Activity profiles of soccer players during the 2010 world cup. Journal of Human Kinetics. 2013; 38: 201-211.

[4] Kelly DM, Strudwick AJ, Atkinson G, Drust B, Gregson W. Quantification of training and match-load distribution across a season in elite English Premier League soccer players. Science and Medicine in Football. 2020; 4: 59-67.

[5] Tierney PJ, Young A, Clarke ND, Duncan MJ. Match play demands of 11 versus 11 professional football using Global Positioning System tracking: Variations across common playing formations. Human Movement Science. 2016; 49: 1-8.

[6] Malone JJ, Di Michele R, Morgans R, Burgess D, Morton JP, Drust B. Seasonal training-load quantification in elite English premier league soccer players. International Journal of Sports Physiology and Performance. 2015; 10: 489-497.

[7] Stevens TGA, de Ruiter CJ, Twisk JWR, Savelsbergh GJP, Beek PJ. Quantification of in-season training load relative to match load in professional Dutch Eredivisie football players. Science and Medicine in Football. 2017; 1: 117-125.

[8] Anderson L, Orme P, Di Michele R, Close GL, Morgans R, Drust B, et al. Quantification of training load during one-, two- and three-game week schedules in professional soccer players from the English Premier League: implications for carbohydrate periodisation. Journal of Sports Sciences. 2016; 34: 1250-1259.

[9] Gabbett TJ, Nassis GP, Oetter E, Pretorius J, Johnston N, Medina D, et al. The athlete monitoring cycle: a practical guide to interpreting and applying training monitoring data. British Journal of Sports Medicine. 2017; 51: 1451-1452.

[10] Haddad M, Stylianides G, Djaoui L, Dellal A, Chamari K. SessionRPE Method for Training Load Monitoring: Validity, Ecological Usefulness, and Influencing Factors. Frontiers in Neuroscience. 2017; 11: 612 .

[11] Foster C. Monitoring training in athletes with reference to overtraining syndrome. Medicine and Science in Sports and Exercise. 1998; 30: 1164-1168.

[12] Heath EM. Borg's Perceived Exertion and Pain Scales. Medicine\& Science in Sports \& Exercise. 1998; 30: 1461.

[13] Gabbett TJ. The training-injury prevention paradox: should athletes be training smarter and harder? British Journal of Sports Medicine. 2016; 50: 273-280

[14] Foster C, Rodriguez-Marroyo JA, de Koning JJ. Monitoring Training Loads: the Past, the Present, and the Future. International Journal of Sports Physiology and Performance. 2017; 12: S22-S28.

[15] Kasper K. Sports Training Principles. Current Sports Medicine Reports. 2019; 18: 95-96.

[16] McLaren SJ, Macpherson TW, Coutts AJ, Hurst C, Spears IR, Weston M. The Relationships between Internal and External Measures of Training Load and Intensity in Team Sports: a Meta-Analysis. Sports Medicine. 2018; 48: 641-658.

[17] Vanrenterghem J, Nedergaard NJ, Robinson MA, Drust B. Training Load Monitoring in Team Sports: a Novel Framework Separating Physiological and Biomechanical Load-Adaptation Pathways. Sports Medicine. 2017; 47: 2135-2142.

[18] Buchheit M. Monitoring accelerations with GPS in football: time to slow Address for correspondence. International Journal of Sports Physiology and Performance. 2013; 442-445.

[19] Jaspers A, Kuyvenhoven JP, Staes F, Frencken WGP, Helsen WF, Brink MS. Examination of the external and internal load indicators' association with overuse injuries in professional soccer players. Journal of Science and Medicine in Sport. 2018; 21: 579-585.

[20] Akenhead R, Nassis GP. Training Load and Player Monitoring in High-Level Football: Current Practice and Perceptions. International Journal of Sports Physiology and Performance. 2016; 11: 587-593.

[21] Cummins C, Orr R, O'Connor H, West C. Global positioning systems (GPS) and microtechnology sensors in team sports: a systematic review. Sports Medicine. 2013; 43: 1025-1042.

[22] Clemente FM, Silva R, Castillo D, Arcos AL, Mendes B. Weekly Load 
Variations of Distance-Based Variables in Professional Soccer Players: A Full-Season Study. International Journal of Environmental Research and Public Health. 2020; 17: 3300.

[23] Clemente F, Silva R, Ramirez-Campillo R, Afonso J, Mendes B, Chen Y. Accelerometry-based variables in professional soccer players: Comparisons between periods of the season and playing positions. Biology of Sport. 2020; 37: 389-403.

[24] Clemente FM, Rabbani A, Conte D, Castillo D, Afonso J, Truman Clark CC, et al. Training/Match External Load Ratios in Professional Soccer Players: A Full-Season Study. International Journal of Environmental Research and Public Health. 2019; 16: 3057.

[25] Akenhead R, Harley JA, Tweddle SP. Examining the External Training Load of an English Premier League Football Team with Special Reference to Acceleration. Journal of Strength and Conditioning Research. 2016; 30: 2424-2432.

[26] Guerrero-Calderón B, Klemp M, Morcillo JA, Memmert D. How does the workload applied during the training week and the contextual factors affect the physical responses of professional soccer players in the match? International Journal of Sports Science \& Coaching. 2021; 0: $1-10$.

[27] Guerrero-Calderón B, Klemp M, Castillo-Rodriguez A, Morcillo JA, Memmert D. A New Approach for Training-load Quantification in Elite-level Soccer: Contextual Factors. International Journal of Sports Medicine. 2020.

[28] Ingebrigtsen J, Dalen T, Hjelde GH, Drust B, Wisløff U. Acceleration and sprint profiles of a professional elite football team in match play. European Journal of Sport Science. 2015; 15: 101-110.

[29] Foster C, Florhaug JA, Franklin J, Gottschall L, Hrovatin LA, Parker $\mathrm{S}$, et al. A new approach to monitoring exercise training. Journal of Strength and Conditioning Research. 2001; 15: 109-115.

[30] Foster C. Monitoring training in athletes with reference to overtraining syndrome. Medicine and Science in Sports and Exercise. 1998; 30: 1164-1168.

[31] Beato M, Coratella G, Stiff A, Iacono AD. The Validity and betweenUnit Variability of GNSS Units (STATSports Apex 10 and $18 \mathrm{~Hz}$ ) for Measuring Distance and Peak Speed in Team Sports. Frontiers in
Physiology. 2018; 9: 1288.

[32] Beato M, Devereux G, Stiff A. Validity and Reliability of Global Positioning System Units (STATSports Viper) for Measuring Distance and Peak Speed in Sports. Journal of Strength and Conditioning Research. 2018; 32: 2831-2837.

[33] Batterham AM, Hopkins WG. Making meaningful inferences about magnitudes. International Journal of Sports Physiology and Performance. 2006; 1: 50-57.

[34] Clemente FM, Owen A, Serra-Olivares J, Nikolaidis PT, van der Linden CMI, Mendes B. Characterization of the Weekly External Load Profile of Professional Soccer Teams from Portugal and the Netherlands. Journal of Human Kinetics. 2019; 66: 155-164.

[35] Springham M, Williams S, Waldron M, Strudwick AJ, Mclellan C, Newton RU. Prior workload has moderate effects on high-intensity match performance in elite-level professional football players when controlling for situational and contextual variables. Journal of Sports Sciences. 2020; 38: 2279-2290.

[36] Paul DJ, Bradley PS, Nassis GP. Factors affecting match running performance of elite soccer players: shedding some light on the complexity. International Journal of Sports Physiology and Performance. 2015; 10: 516-519.

[37] Oliva-Lozano JM, Rojas-Valverde D, Gómez-Carmona CD, Fortes V, Pino-Ortega J. Worst case scenario match analysis and contextual variables in professional soccer players: a longitudinal study. Biology of Sport. 2020; 37: 429-436.

[38] Duhig S, Shield AJ, Opar D, Gabbett TJ, Ferguson C, Williams M. Effect of high-speed running on hamstring strain injury risk. British Journal of Sports Medicine. 2016; 50: 1536-1540.

[39] Malone S, Hughes B, Doran DA, Collins K, Gabbett TJ. Can the workload-injury relationship be moderated by improved strength, speed and repeated-sprint qualities? Journal of Science and Medicine in Sport. 2019; 22: 29-34.

[40] Malone S, Owen A, Mendes B, Hughes B, Collins K, Gabbett TJ. Highspeed running and sprinting as an injury risk factor in soccer: can welldeveloped physical qualities reduce the risk? Journal of Science and Medicine in Sport. 2018; 21: 257-262. 